\title{
A Model for Damage Spreading with Damage Healing: Monte Carlo Study of the two Dimensional Ising Ferromagnet.
}

\author{
M. Leticia Rubio Puzzo and Ezequiel V. Albano \\ Instituto de Investigaciones Fisicoquímicas Teóricas y Aplicadas (INIFTA), UNLP, CONICET, \\ Casilla de Correo 16 Sucursal 4, (1900) La Plata, Argentina.
}

\begin{abstract}
An Ising model for damage spreading with a probability of damage healing $(q=1-p)$ is proposed and studied by means of Monte Carlo simulations. In the limit $p \rightarrow 1$ the new model is mapped to the standard Ising model. It is found that, for temperatures above the Onsager critical temperature $\left(T_{C}\right)$, there exist a no trivial finite value of $p$ that sets the critical point $\left(p_{c}\right)$ for the onset of damage spreading. It is found that $p_{c}$ depends on $T$, defining a critical curve at the border between damage spreading and damage healing. Transitions along such curve are found to belong to the universality class of directed percolation. The phase diagram of the model is also evaluated showing that for large $T$ one has $p_{c} \propto\left(T-T_{C}\right)^{\alpha}$, with $\alpha=1$. Within the phase where the damage remains active, the stationary value of the damage depends lineally on both $p-p_{c}$ and $T-T_{C}$.
\end{abstract}

Keywords: Damage propagation in magnetic materials, Stochastic processes, Monte Carlo numerical simulations.

PACS numbers: 02.50.Ey, 05.40.-a, 75.10.Hk, 05.10.Ln 


\section{Introduction}

The damage spreading method has been widely used to study the critical properties of Ising-like systems [1, 2, 3, [4, 5, 6, 7, 8] as well as to spin glass [9, 10]. The method is based on the synchronous Monte Carlo update of two distinct spin configurations that are evolving from an almost identical initial state [11]. Since the Ising model lacks of an intrinsic dynamic, it has to be chosen a particular one. Among others, the more frequently used are Glauber and heat-bath dynamics. In contrast to usual statistical Monte Carlo studies, damage spreading results depends on the used dynamic [11. This behavior has been used to evaluate the dynamical exponent $z$ of the 2D and 3D Ising models with different dynamics [3, 12, 13. Also, very recently, it has been shown that damage spreading is a powerful and useful technique for the numerical study of the role of the interfaces between magnetic domains on the propagation of perturbations in magnetic materials [14, 15]. Within this context, we have reported that the presence of interfaces act as a "catalyst" of the damage in at least two different ways: speeding up the propagation and causing an enhancement of the total damaged area [14, 15].

The basic idea for the implementation of the damage spreading method [11 is to start from an equilibrium configuration of the system at temperature $T$, which is generically called $S^{A}(T)$. Subsequently, a very small perturbation is applied to that configuration in order to obtain a new one, i.e. the so called perturbed configuration $S^{B}(T)$. Usually the perturbed configuration is obtained by flipping a small number of spins of the unperturbed configuration. Then one has to study the time evolution of the perturbation in order to investigate under which conditions such a small perturbation will grow up indefinitely or eventually (hopefully) it will vanish and become healed. In order to follow the time evolution of the perturbation an useful method is to measure the "Hamming distance" or damage between the unperturbed and the perturbed configurations. The total damage $D(t)$ is defined as the fraction of spins with different orientations, that is

$$
\left.D(t)=\frac{1}{2 N} \sum_{l}^{N} \mid S_{l}^{A}(t, T)-S_{l}^{B}(t, T)\right] \mid,
$$

where the summation runs over the total number of spins $\mathrm{N}$ and the index $l(1 \leq l \leq N)$ is the label that identifies the spins of the configurations. Starting from a vanishing small perturbation $D(t=0) \rightarrow 0$ one can expects at least two scenarios, namely: a) $D(t \rightarrow \infty) \rightarrow 0$ and the perturbation is irrelevant because it become healed and b) $D(t \rightarrow \infty)$ goes to some well defined finite value. In the latter, frequently undesired case, the perturbation is relevant because it can not be healed out. For further details see the review [11].

The aim of this work is propose and study a model for damage spreading with damage healing, which is based in the two dimensional $(2 D)$ Ising model. It is well known that the $2 D$ Ising magnet undergoes a second-order orderdisorder transition when the temperature is raised from a relatively low initial value. The location of the critical point is know exactly and it is given by the so called Onsager critical temperature $\frac{k T_{C}}{J}=2.269 \ldots$. According to previous studies of damage spreading using Glauber dynamics it is known that there is a critical damage temperature given by $T_{D} \cong 0.992 T_{C}$ [16, 17, such as for $T>T_{D}$ the damage spreads out over the whole sample while for $T<T_{D}$ the damage becomes healed after some finite time. Consequently, the proposed model, that incorporates a healing probability, is suitable for the study of damage spreading above $T_{D}$, as well as to gain insight on the robustness of the damage behavior in the Ising model.

The manuscript is organized as follows: in Section II we propose the model and describe the numerical procedure for the simulation of damage spreading. Section III is devoted to the presentation and discussion of the results, while our conclusions are stated in Section IV.

\section{A Model for Damage Spreading with Healing and the Monte Carlo Simulation Method.}

The model is based on the $2 D$ ferromagnetic Ising model, which for a square lattice of side $L$ can be described by the following Hamiltonian $H$ :

$$
H=-J \sum_{<i j, m n>}^{L, L} \sigma_{i j} \sigma_{m n}
$$


where $\sigma_{i j}$ is the spin variable, corresponding to the site of coordinates $(\mathrm{i}, \mathrm{j})$, that may assume two different values, namely $\sigma_{i j}= \pm 1, J>0$ is the coupling constant of the ferromagnet and the summation of (2) runs over all the nearest-neighbor pairs of spins.

For the purpose of the simulations, periodic boundary conditions are always used. Furthermore, we have used the Glauber dynamics. In order to implement this dynamic a randomly selected spin is flipped with probability $p($ flip $)$ given by:

$$
p(\text { flip })=\frac{\exp (-\beta \cdot \triangle H)}{1+\exp (-\beta \cdot \triangle H)}
$$

where $\triangle H$ is the difference between the energy of the would-be new configuration and the old configuration, and $\beta=1 / k T$ is the usual Boltzmann factor. The temperature is measured in units of the Onsager critical temperature of the $2 D$ Ising model.

In order to study the time evolution of the damage spreading a meaningful definition of the Monte Carlo time step (mcs) is necessary. For this purpose the standard definition is adopted according to that during one mcs all $L \times L$ spins of the sample are flipped once, in the average.

For the practical implementation of equation (11), first an equilibrium configuration (say configuration $S_{e}^{A}$ ) is generated. For this purpose one starts from an initial configuration with all $N=L \times L$ spins oriented at random. The application of the Glauber dynamics leads to the desired equilibrium configuration after $10^{4} \mathrm{mcs}$. Subsequently, a fully damaged replica $S_{e}^{B}$ of such configuration is created, such as $S_{e}^{B}$ is the mirror image of $S_{e}^{A}$ and consequently $D(0)=1$. Of course, due to the spin flip symmetry, the replica configuration is also equilibrated.

In standard damage spreading studies, the standard Monte Carlo procedure is then applied to both configurations, where the same sites are randomly selected and the same random numbers are used in both systems in order to perform the updates. For the purpose of the proposed model we first define the healing probability $q$ and we used the same method but according the following rules:

i) A sample site of coordinates $(i, j)$ is selected at random. Then,

iia) If $S^{A}(i, j)=S^{B}(i, j)$, which means that the selected site is not damaged, the Glauber dynamics is followed according to the standard procedure [11]. However,

iib) If $S^{A}(i, j) \neq S^{B}(i, j)$, i.e. for a damaged site, a new random number $h$ is generated and one proceeds as follows:

iib1) If $h<q$, the damage is healed, so that one sets $S^{A}(i, j)=S^{B}(i, j)$. However,

iib2. If $h \geq q$, the standard dynamics is applied.

According to the defined rules and taking $p=1-q$, one has that for $p \rightarrow 0$, the damage would become healed for all temperatures, while in the limit $p \rightarrow 1$, the usual damage spreading dynamics in the Ising model is recovered.

Using the above described procedure, we have followed the time evolution of the damage $D(t)$, which is evaluated according to equation (1), for different values of $p, 1.1 \leq T \leq 50$ and the lattice size $50 \leq L \leq 1000$. Simulations are stopped when the damage is healed, otherwise they are performed up to $t=65000$ mcs.

\section{Results and discussion}

Figure 1 shows $\log -\log$ plots of $D(t)$ versus $t$ obtained at $T=2$ for different values of $p$. It is found that: i) for $p>p_{c}$ the damage becomes healed for finite times and the log-log plots of $D(t)$ versus $t$ exhibit a downward curvature. ii) For $p<p_{c}$ the damage quickly propagates and the log-log plots of $D(t)$ versus $t$ show that the damage reaches a stationary value. Finally, just at $p=p_{c}=0.1895(5)$ the damage decays according to a power law. So, the change of the curvature of the plots shown in figure 1 allows us to identify the critical probability $p_{c}$ for damage spreading.

The straight line observed in figure 1 for $p_{c}$ suggests a power-law behavior given by

$$
D(t) \propto t^{-\delta}
$$

where $\delta$ is an exponent. The best fit of the data gives $\delta=0.450 \pm 0.005$. Similar plots obtained for $T>T_{C}$ (not shown here for the sake of space) exhibit the same behavior as that observed in figure 1 and the typical slope, averaged within the range $2 \leq T \leq 50$, is given by $\delta=0.45 \pm 0.01$.

On the other hand, for $p>p_{c}$ figure 1 shows a fast decay of the damage. As suggested by the study of spreading behavior [18], we proposed an exponential decay given by 


$$
D(t) \propto \exp (-t / \tau)
$$

where $\tau$ is the characteristic time of decay.

In order to check the scaling Ansatz given by equation (5), figure 2 shows log-lineal plots of $D(t)$ versus $t$ obtained for $p>p_{c}$. The obtained straight lines for the long time behavior allow us to evaluate the dependence of $\tau$ on $p$.

It is found that $\tau$ increases when approaching the critical probability. Furthermore, plots performed at different temperatures (no shown here for the sake of space) also exhibit the same behavior, independent of $T$. Again, using the spreading formalism [18], the following power-law can be proposed

$$
\tau \propto\left(p-p_{c}\right)^{-\nu_{\|}}
$$

where $\nu_{\|}$is the correlation length exponent for damage propagation along the time direction. Figure 3 shows log-log plots of $\tau$ versus $p-p_{c}$ obtained at different temperatures. The typical averaged value resulting from the fits is given by $\nu_{\|}=1.25(5)$.

It is worth mentioning that our estimations for the exponents, given by $\delta=0.45(1)$ and $\nu_{\|}=1.25(5)$ are in agreement with the well known critical exponents of the universality class of Directed Percolation (DP), namely $\delta^{D P}=0.4505(10)$ [19] and $\nu_{\|}^{D P}=1.295(6)$ [20]. So, these results strongly suggest that the the proposed model of damage spreading with damage healing belongs to the DP universality class. This statement is further supported by the fact that the limit $p \rightarrow 1$ corresponds to the well known Ising model where the damage spreading transition is known to lie within the DP universality class [16, 17.

On the other hand, as follows from figure 1 , for $p<p_{c}^{D}$ and after a short decay period $\left(t \approx 10^{2} \mathrm{mcs}\right)$, the damage becomes stabilized (or saturated) with typical average values $D_{\text {sat }}$ that depend on $p$. This trend has been checked by means of extensive simulations run up to $t=10^{6} \mathrm{mcs}$. Furthermore, we have also checked that the stationary values of $D_{\text {sat }}$ exhibit negligible finite-size effects for $L \geq 200$. Figure 4 shows the dependence of $D_{\text {sat }}$ on $\left(p_{c}^{D}-p\right)$ in a lineal-lineal plot. It follows that $D_{\text {sat }}$ increases lineally as a function of $\left(p_{c}^{D}-p\right)$, and that the slope $F(T)$ depends on temperature. This assumption is valid only in the limit of $p \rightarrow p_{c}^{D}$. Accordingly, we propose

$$
D_{\text {sat }}=D_{0}+F(T)\left(p_{c}^{D}-p\right),
$$

where $D_{0} \approx 0.05$ is almost a constant independent of $T$ (see figure 4 ). The slope of the lines can be obtained by fitting the data of figure 4 and it is found that $F(T)$ also depends lineally with $T$ (see figure 5) according to

$$
F(T)=F_{0}+A\left(\frac{T-T_{C}}{T_{C}}\right)
$$

where $F_{0} \approx 0$ and $A \approx 1.5$ are also constants.

So, by inserting equation (8) into equation (7), one obtains

$$
D_{\text {sat }}=D_{0}+\left(F_{0}+A \frac{T-T_{C}}{\left.T_{C}\right)}\right) \cdot\left(p-p_{c}^{D}\right)
$$

that gives the full dependence of $D_{\text {sat }}$ on both $p$ and $T$. The scaling plot suggested by equation (9) holds acceptably as shown in figure 6,

Performing plots of the time evolution of the damage using different values of $p$ (as shown in figure 11) and changing $T$, it is finally possible to evaluate the phase diagram of the model, i.e. a plot of $p_{c}^{D}$ as a function of $\left(T-T_{C}\right) / T_{C}$, as shown in figure 7 . It is found that $p_{c}^{D} \rightarrow 0$ for very large values of $T$, as expected from the definition of the model. For the asymptotic decay of $p_{c}^{D}$ we propose

$$
p_{c}^{D} \propto\left(\frac{T-T_{C}}{T_{C}}\right)^{-\alpha}
$$

where the best fit of the data, within the range $T \gg 1.5 T_{C} \sim 3.4 J$, gives $\alpha=1$ for the exponent of equation (10). It is worth mentioning that, for the lattices used in the simulations, the phase diagram exhibits negligible deviations due to finite size effects, as shown in figure 7 . On the other hand, close to $T_{C}$, it is observed that $p_{c}^{D}$ reaches a maximum value of the order of $p_{c}^{D} \sim 0.22$ for $T=1.5 T_{C} \sim 3.4 \mathrm{~J}$. We have carefully checked that this behavior, 
which remains to be understood, is not due to the operation of finite size effects. Finally, let us remark that all phase transitions at the critical curve at the border between damage healing and damage spreading, shown in figure 7, belong to the universality class of directed percolation.

\section{Conclusions}

Based on the fact that in the 2D Ising model the damage is not healed above the critical temperature, we proposed a model that introduces a healing probability. It is found that for $T>T_{C}$ the damage becomes healed for critical values of $p$. The phase diagram of the model is obtained and it is shown that the transitions between states of damage spreading and healing belong to the universality class of directed percolation in $(2+1)$-dimensions. Our results thus support the conjecture that damage spreading transitions are generically in the universality class of directed percolation [16], provided the fact that such transitions do not coincide with the critical point of the undamaged system.

\section{References}

[1] B. Derrida, D. Stauffer, Europhys. Lett. 2, (1986) 739.

[2] H. E. Stanley, D. Stauffer, J. Kertesz, H. J. Herrmann, Phys. Rev. Lett. 59, (1987) 2326.

[3] P. Grassberger, Physica A 214, (1995) 547.

[4] F. Montani, E. V. Albano, Phys. Lett. A 202, (1995) 256.

[5] F. Wang, N. Hatano, M. Suzuki, J. Phys. A (Math. and Gen.) 28, (1995) 4543.

[6] T. Vojta, J. Phys. A (Math. and Gen.) 30, (1997) L7.

[7] A. V. Lima, M. L. Lyra, U. M. S. Costa, J. Appl. Phys. 81, (1997) 3983.

[8] A. V. Lima, M. L. Lyra, U. M. S. Costa, J. Mag. Magn. Mat. 171, (1997) 329.

[9] B. Derrida, G. Weisbuch, Europhys. Lett. 4, (1987) 657.

[10] C. Argolo, A. M. Mariz, S. M. Miyazima, Physica A 264, (1999) 142.

[11] H. J. Herrmann, in "The Monte Carlo method in condensed matter physics", Ed. K. Binder (Springer, Berlin, 1992).

[12] F. Wang, M. Suzuki, Physica A 223, (1996) 34.

[13] D. Stauffer, J. Phys. A (Math. and Gen.) 26, (1993) L599.

[14] M. L. Rubio Puzzo and E. V. Albano. J. Mag. Mag. Matt. 241, 110 (2002).

[15] M. L. Rubio Puzzo and E. V. Albano. Phys. Rev. B. 66, 104409 (2002).

[16] P. Grassberger, J. Phys. A (Math. and Gen.) 28, (1995) L67.

[17] P. Grassberger, J. Stat. Phys. 79, (1995) 13.

[18] P. Grassberger and A. de la Torre, Ann. Phys. (NY). 122, 373 (1979).

[19] C. A. Voigt and R. M. Ziff, Phys. Rev. E. 56, R6241 (1997).

[20] P. Grassberger and Y. Zhang, Physica A 224, 169 (1996). 


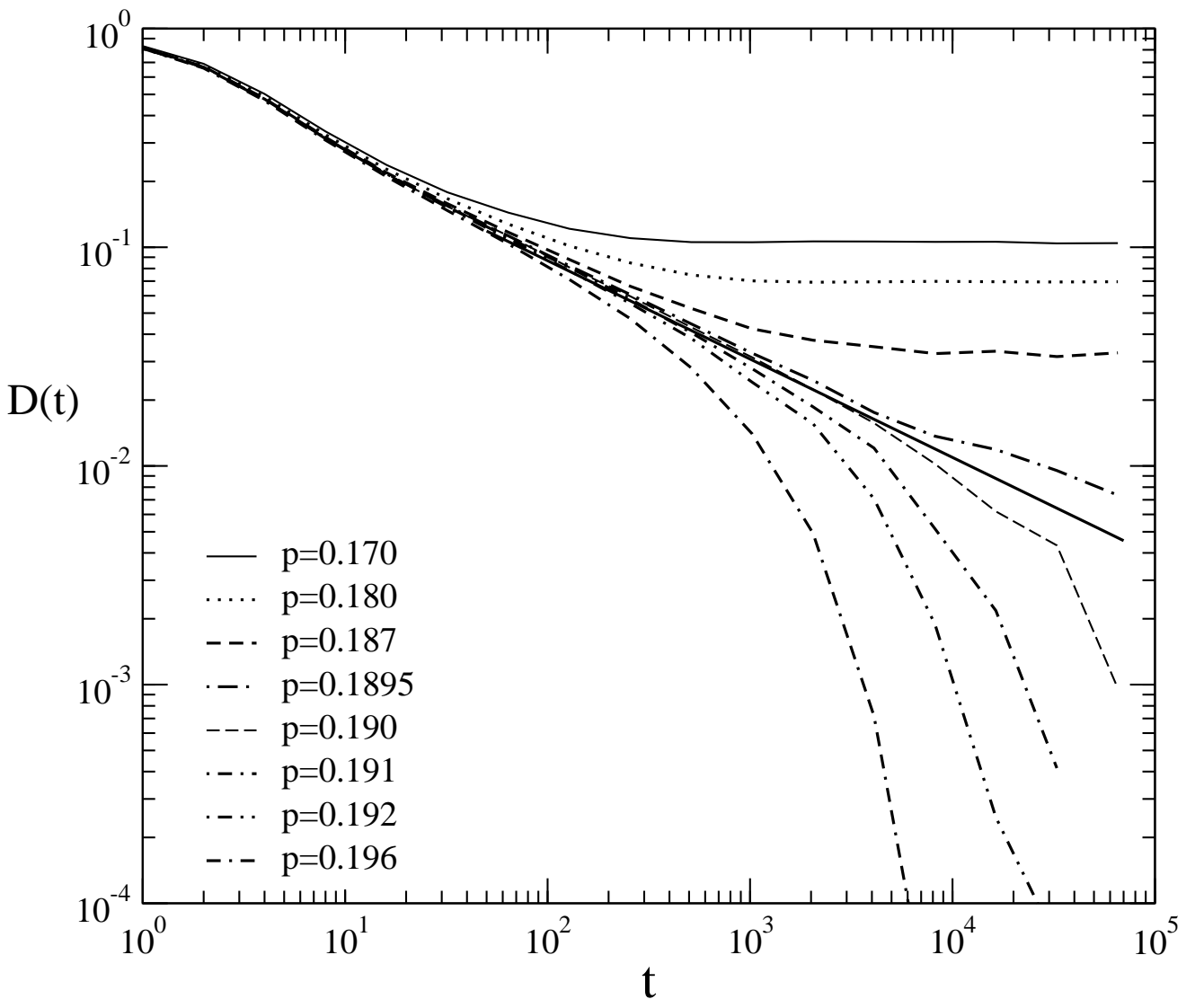

Figure 1: Log-log plots of the damage $(D(t))$ versus time $t$. Data obtained using lattices of side $L=400$, for $T=2.0 T_{C}$ and taking different values of $p$, as listed in the figure. The full line has slope $-\delta=-0.45$ (see equation (41) ). 


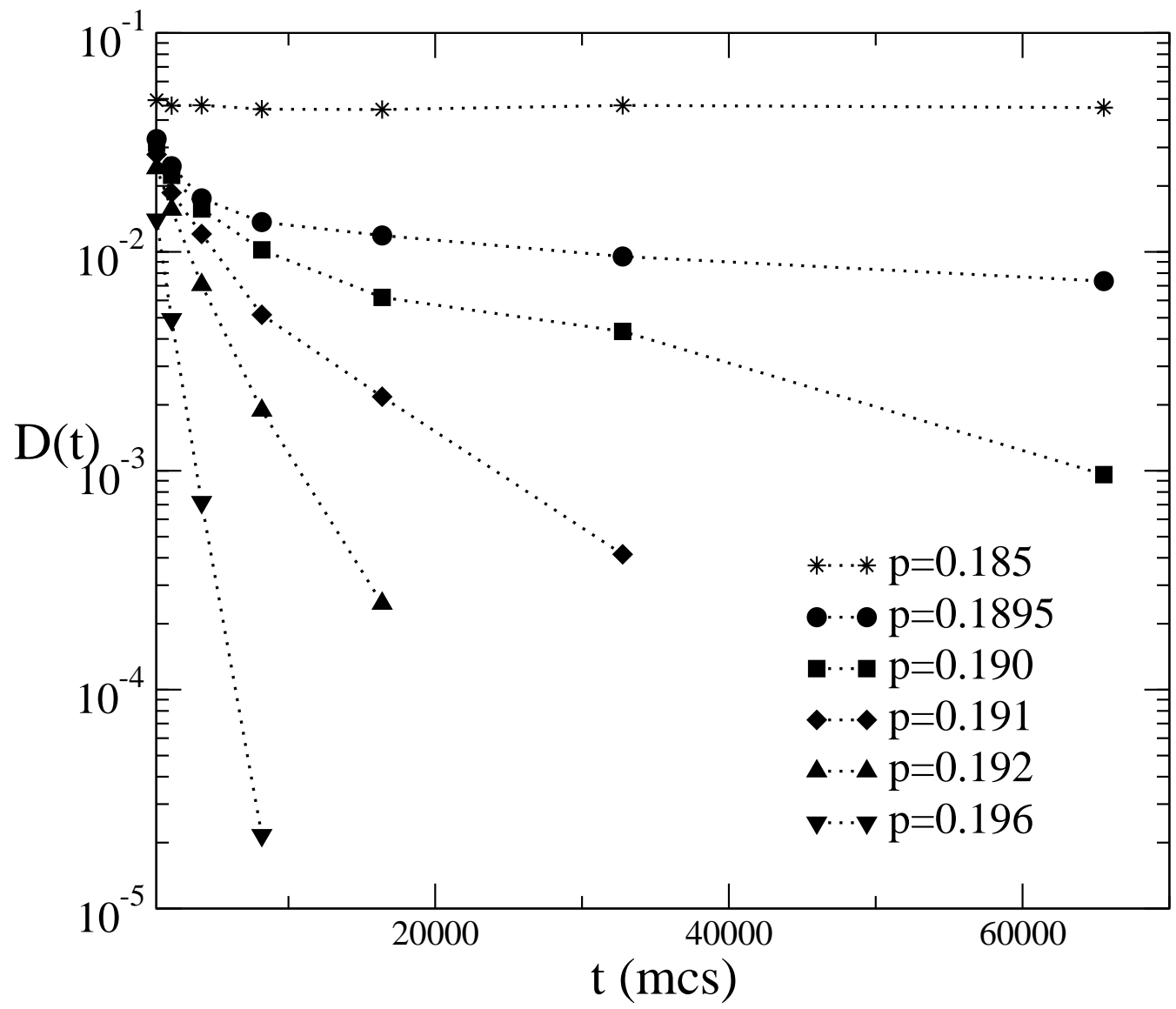

Figure 2: Log-lineal plots of the damage $(D(t))$ versus $t$ for data corresponding to $p>p_{c}^{D}$ and obtained taken $L=400$ and $T=2.0 T_{C}$. 


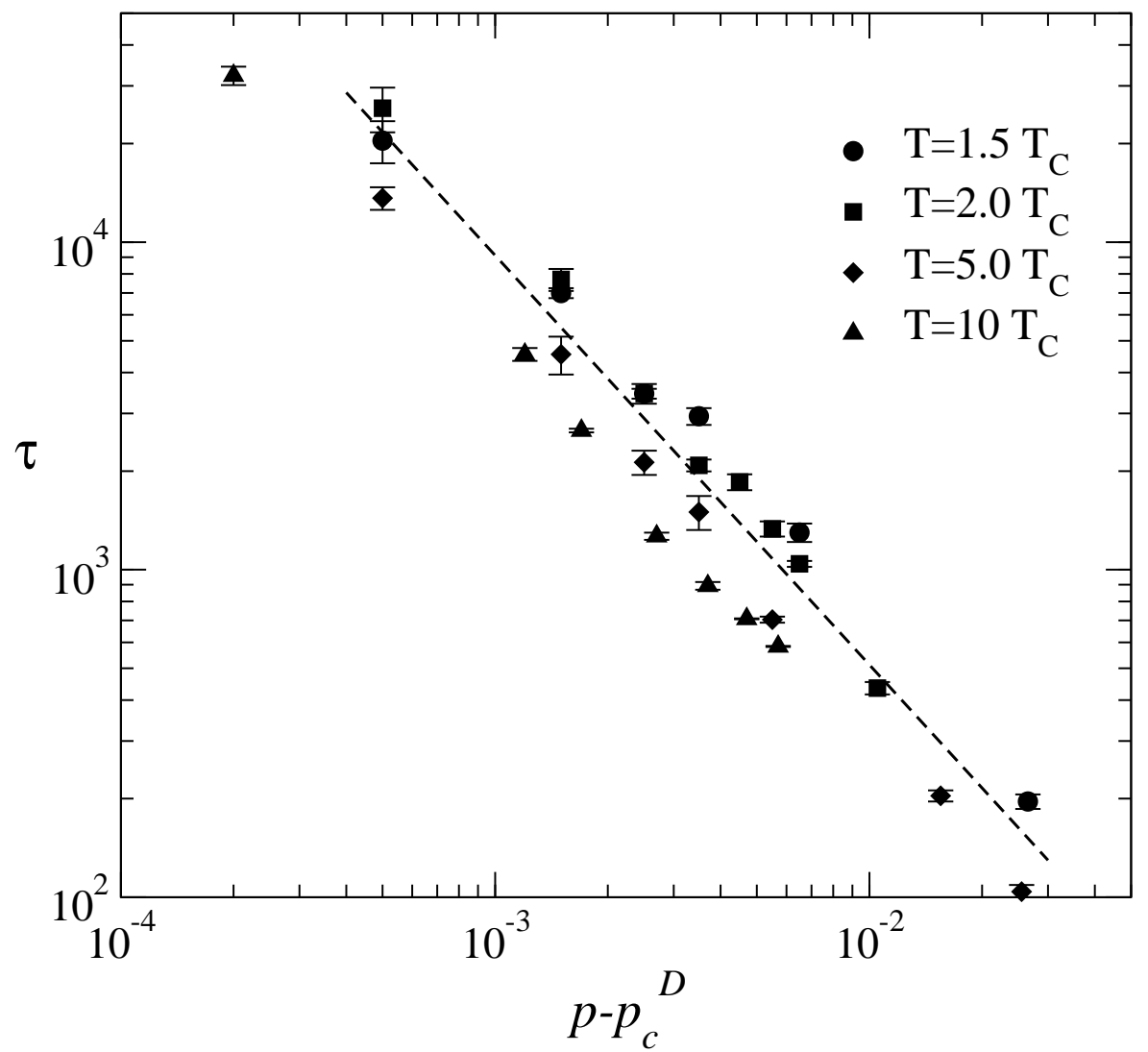

Figure 3: $\log -\log$ plot of characteristic time for damage healing $\tau$, obtained by fitting equation (5) to data as shown in figure 2, versus $p-p_{c}^{D}$. Data obtained using lattices of size $L=400$ and different temperatures, as listed in the figure. The dashed line, which has slope $\nu_{\|}=1.25$, is the slope obtained averaging over all measured temperatures and has been drawn for the sake of comparison. 


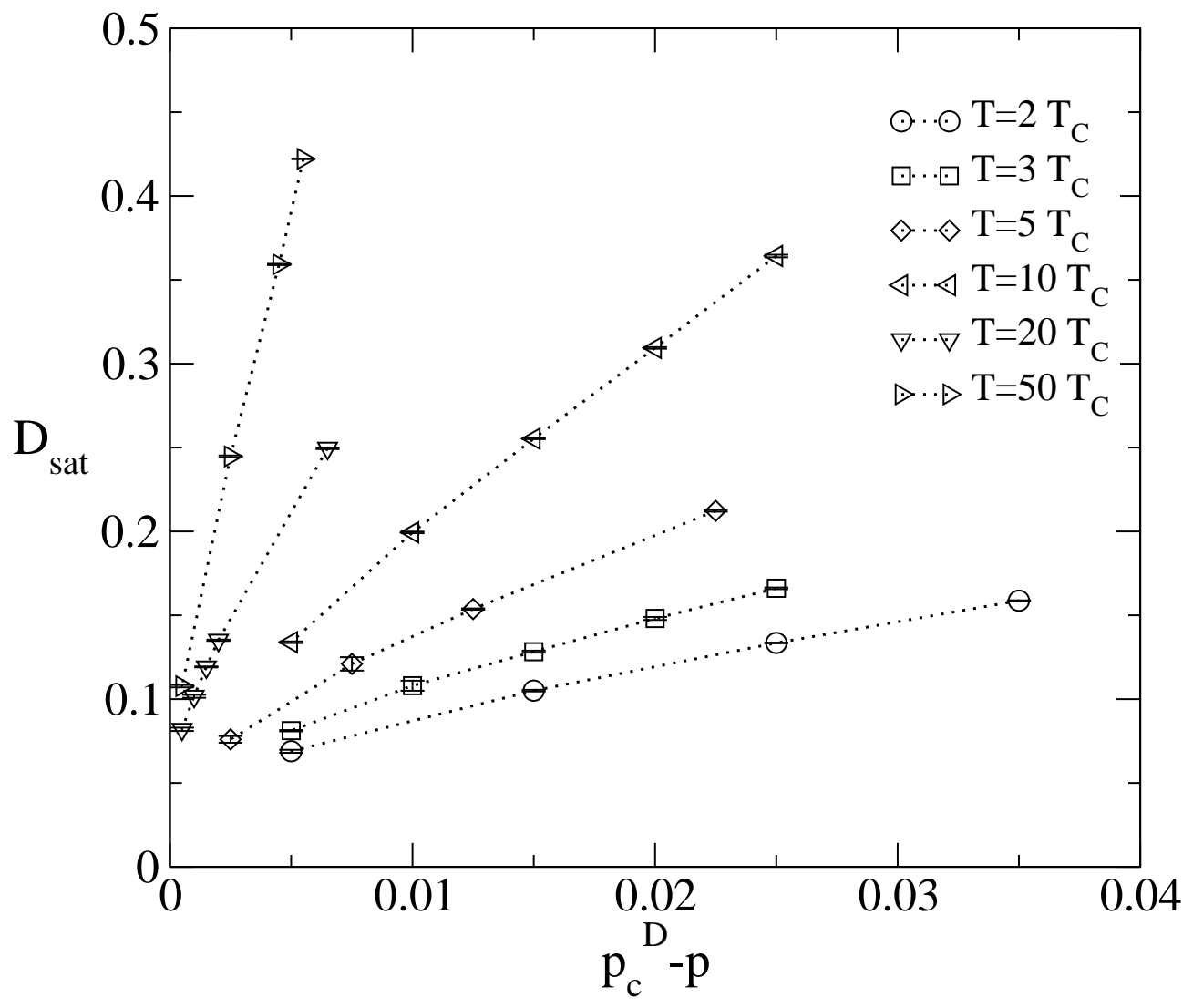

Figure 4: Lineal-lineal plots of $D_{\text {sat }}$ versus $p_{c}^{D}-p$ obtained for different values of $T$, as listed in the figure. 


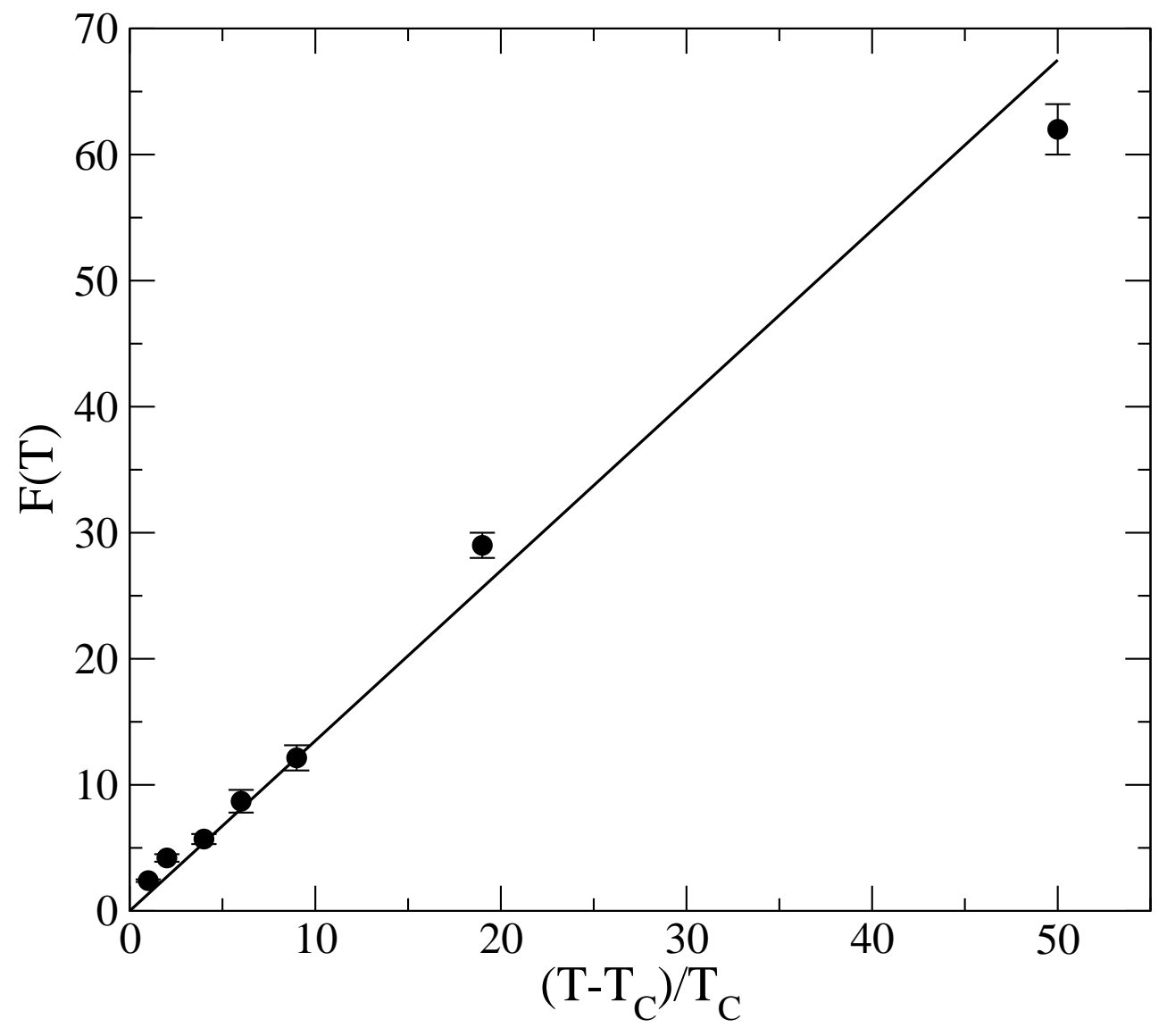

Figure 5: Lineal-lineal plots of the slopes obtained by fitting the data shown in figure 4 by means of equation (7) given by $F(T)$, versus $\left(T-T_{C}\right) / T_{C}$. The straight line with slope $A \approx 1.5$ was obtained by fitting the data according to equation (8) . 


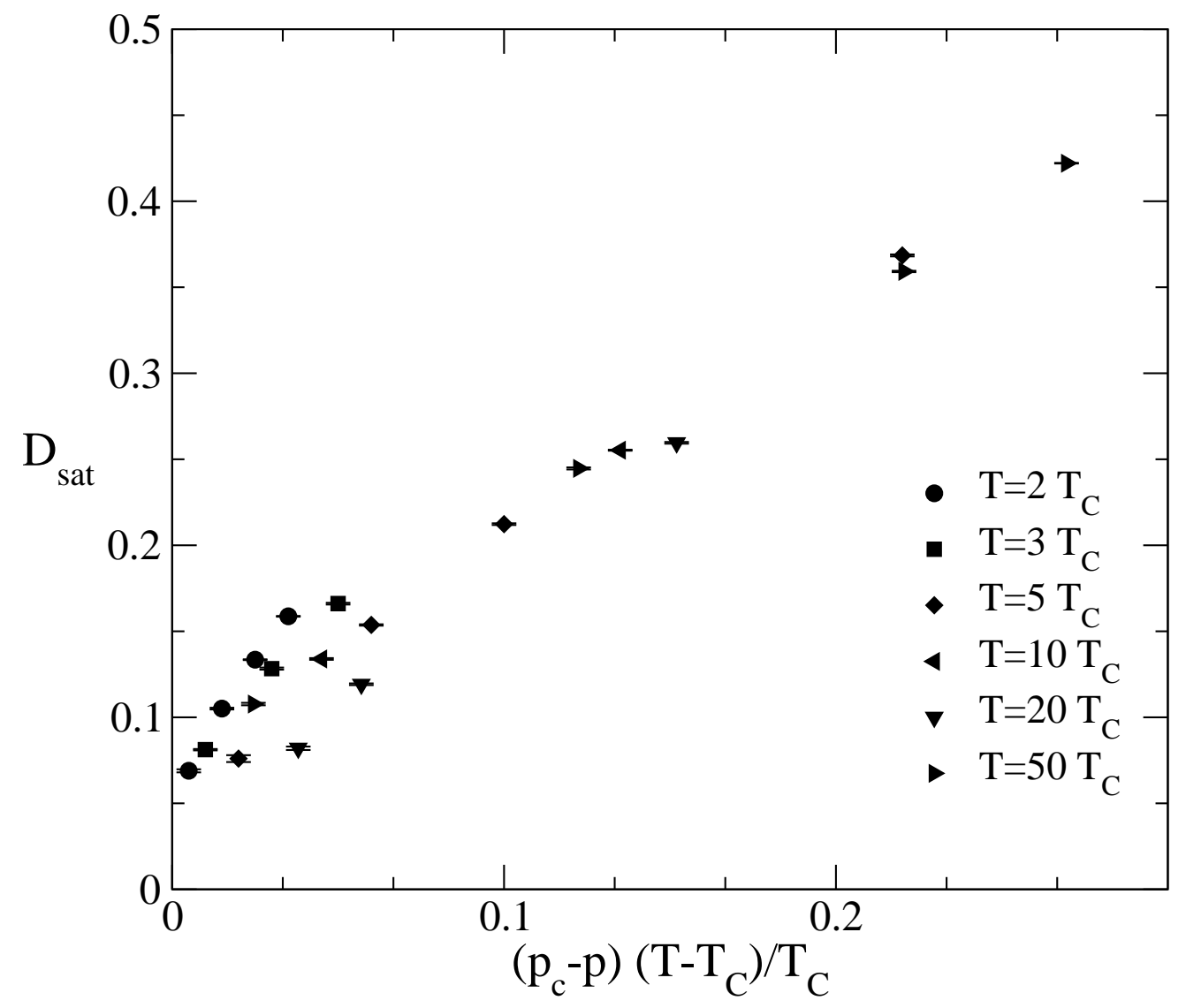

Figure 6: Scaling plot of $D_{\text {sat }}$ according to equation (9), obtained taking $F_{0}=0$. More details in the text. 


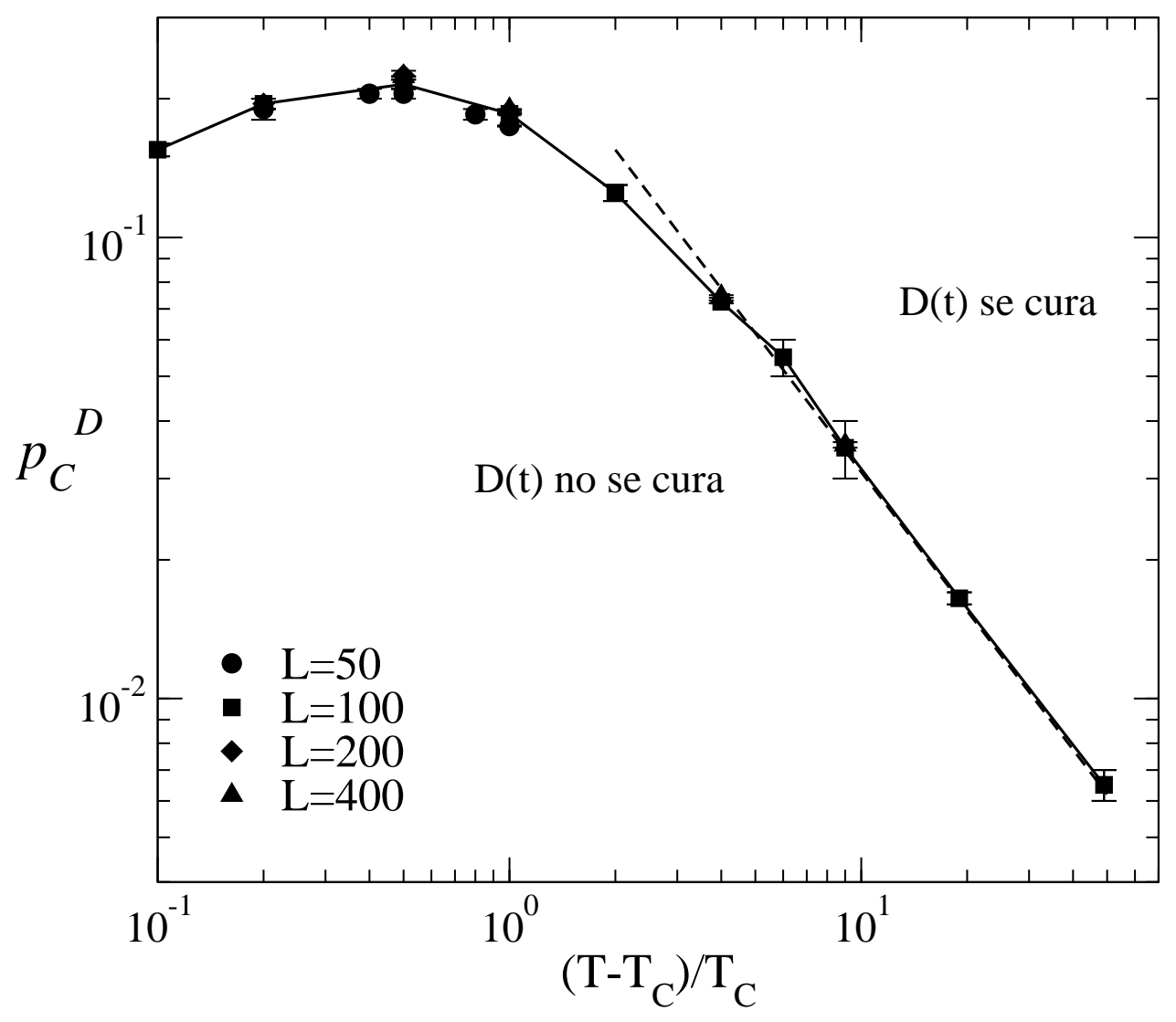

Figure 7: Phase diagram for the proposed model for damage spreading with a probability of damage healing. The full line has been drawn to guide the eyes. The dashed line corresponds to the fit of the data for $T \gg 1.5 T_{C}$ and has slope $-\alpha=-1$. 